\title{
Gamble mode: Resonance contact mode in atomic force microscopy
}

\author{
S. D. O'Connor \\ Division of Chemistry 127-72, California Institute of Technology, Pasadena, California 91125 \\ R. C. Gamble and R. K. Eby \\ Topometrix, Santa Clara, California 95054-1162 \\ J. D. Baldeschwieler \\ Division of Chemistry 127-72, California Institute of Technology, Pasadena, California 91125
}

(Received 25 July 1995; accepted 25 January 1996)

\begin{abstract}
Active noise reduction has been accomplished in atomic force microscopy by applying a high frequency, low amplitude vibration to the cantilever while it is in contact with a surface. The applied excitation $(>200 \mathrm{kHz} ; \sim 1 \mathrm{~nm})$ is acoustically coupled to the tip and dampens the resonance $Q$ factors of the system. The applied frequency is well above the bandwidth of the acquisition system $(50 \mathrm{kHz})$. We call this mode "gamble mode" or "resonance contact." (C) 1996 American Vacuum Society.
\end{abstract}

\section{INTRODUCTION}

Active noise reduction is of interest for any analytical instrument and crucial in certain environments. Mechanical noise can induce unwanted signals in atomic force microscopy measurements, limiting the resolution and scan speed. In this article, a method for reducing mechanical noise in atomic force microscopy (AFM) is demonstrated; this technique is easy to implement with minimal instrumentation.

The use of atomic force microscopy in a dynamic mode was first demonstrated by Martin and co-workers in 1987. ${ }^{1}$ This "resonance noncontact" mode is implemented as follows: the cantilever is vibrated at its first resonance frequency $\left(\omega_{0}\right)$. As the tip approaches the sample, the tipsample interaction produces a force gradient that shifts the resonance frequency of the cantilever:

$$
m^{*} \omega_{\text {new }}^{2}=k_{\text {new }}=k_{0}-\frac{\partial F}{\partial z},
$$

where $k$ is the spring constant, $m^{*}$ is the effective mass, $\omega$ is the resonance frequency, and $z$ is the tip-sample separation. Since the driving frequency $\left(\omega_{0}\right)$ is not equal to the new resonance frequency of the cantilever $\left(\omega_{\text {new }}\right)$, the amplitude of the vibration is lowered and can be used as a feedback signal. This technique effectively negates the lateral force that is present in normal contact mode imaging. However, this strategy requires lock-in electronics and special cantilevers.

Recently, resonant noncontact mode imaging was demonstrated in water with cantilevers having relatively weak spring constants $(0.5 \mathrm{~N} / \mathrm{m}){ }^{2,3}$ Also, ultrasonic detection has been used in conjunction with AFM to detect cantilever movements in the $\mathrm{MHz}$ regime. ${ }^{4}$

In this article, a simple method for reducing low frequency noise in dc AFM experiments is demonstrated. While the cantilever is in contact with the sample, the base of the cantilever is mechanically driven at a high frequency. At certain frequencies, the overall mechanical noise of the system is lowered. No special instrumentation is required for this mode other than a function generator to vibrate the cantilever at high frequency and a piezoceramic to couple the excitation into the cantilever-sample system. This technique is referred to as "resonance contact" or "gamble mode" (GM).

\section{EXPERIMENT}

A Topometrix Discoverer AFM with in-house software was used for the experiments in this article. ${ }^{5,6}$ This system uses an optical lever detection scheme to monitor the cantilever displacement. In order to determine the frequency characteristics of the cantilever motion, the output of the photodiode preamplifier was connected directly to a HP spectrum analyzer. Standard $200 \mu \mathrm{m}$, thin arm triangular cantilevers were used for all of the experiments $\left(k=0.1 \mathrm{~N} / \mathrm{m}, \omega_{0} \sim 20\right.$ $\mathrm{kHz})$.

The software uses a proportional-integral-differential (PID) feedback algorithm to maintain constant cantilever deflection. The feedback parameters determine the response time of the instrument; higher values will produce shorter response times. However, if the gains are increased above a

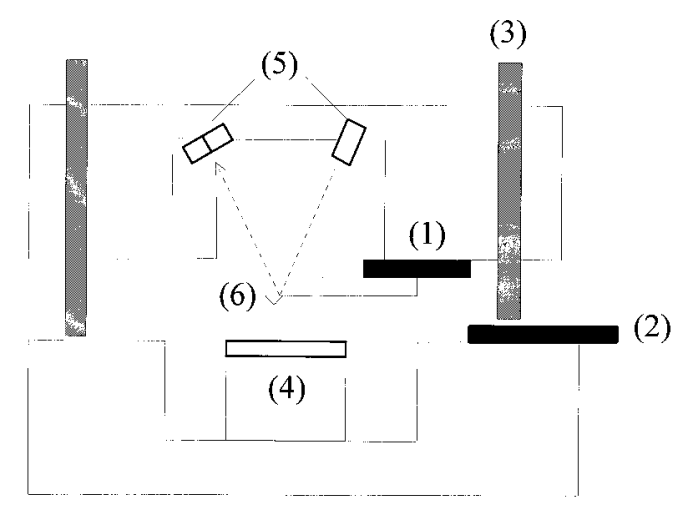

FIG. 1. Schematic of the GM setup. Two piezos can drive the GM frequency: (1) GM piezo A or (2) GM piezo B; (3) approach screws; (4) sample tube scanner; (5) feedback laser and photodiode; (6) cantilever. 


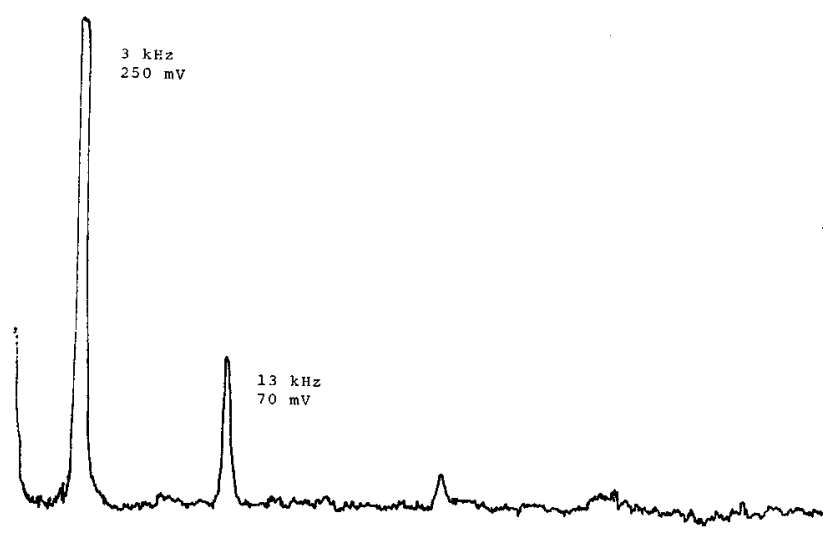

FIG. 2. Typical noise spectrum; the integral gain is set $\sim 8$ times higher than the optimal value; the $3 \mathrm{kHz}$ noise $(\sim 250 \mathrm{mV})$ is due to the feedback parameters being higher than optimal; the $13 \mathrm{kHz}$ noise is a mechanical resonance and is $\sim 70 \mathrm{mV}$.

certain threshold, the feedback loop will oscillate. This threshold is one of the factors limiting the maximum scan speed.

In order to vibrate the cantilever, the tip mounting system was attached directly to a small piezoceramic (labeled GM piezo A in Fig. 1). This baseplate was initially designed to be used in resonance noncontact mode. For the liquid experiments, a piezoceramic was glued between the AFM head that houses the tip/detector and the base that houses the sample. Driving this piezo was also effective at coupling vibrations to the cantilever. This piezo will be referred to as GM piezo B. A function generator was used to apply the driving signal.

\section{RESULTS}

\section{A. Typical noise spectrum}

Figure 2 is a noise spectrum when the cantilever is in contact with a compact disk (CD). Two specific noise peaks are present in this experiment: 3 and $13 \mathrm{kHz}$. The $3 \mathrm{kHz}$ noise changes amplitude as the feedback gains are altered but does not shift in frequency. A change in imaging conditions may shift this frequency slightly. For example, changing the meniscus from oil to water shifts this frequency to $\sim 2.7$ kHz. However, using a new cantilever, sample, or setpoint does not shift this frequency. There is also a mechanical resonance at $13 \mathrm{kHz}$; this noise varies in amplitude and frequency for different tips and samples.

No particular sources of room noise are present between $1-20 \mathrm{kHz}$, within the detection limits of the system. There-

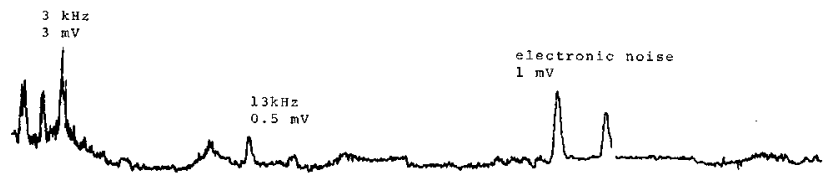

FIG. 3. Noise in Fig. 2 after GM is initiated at $356 \mathrm{kHz}$; notice the new scale. The small peaks above $30 \mathrm{kHz}$ (far right) are electronic noise. fore, the large noise peaks that are observed when in contact must arise from structural modes with very large quality factors $(Q)$.

The noise level in Fig. 2 is actually very high for this instrument; typical ambient noise is $\sim 10 \mathrm{mV}$ total with a baseline $\ll 1 \mathrm{mV}$. However, to demonstrate the power of this

(a)
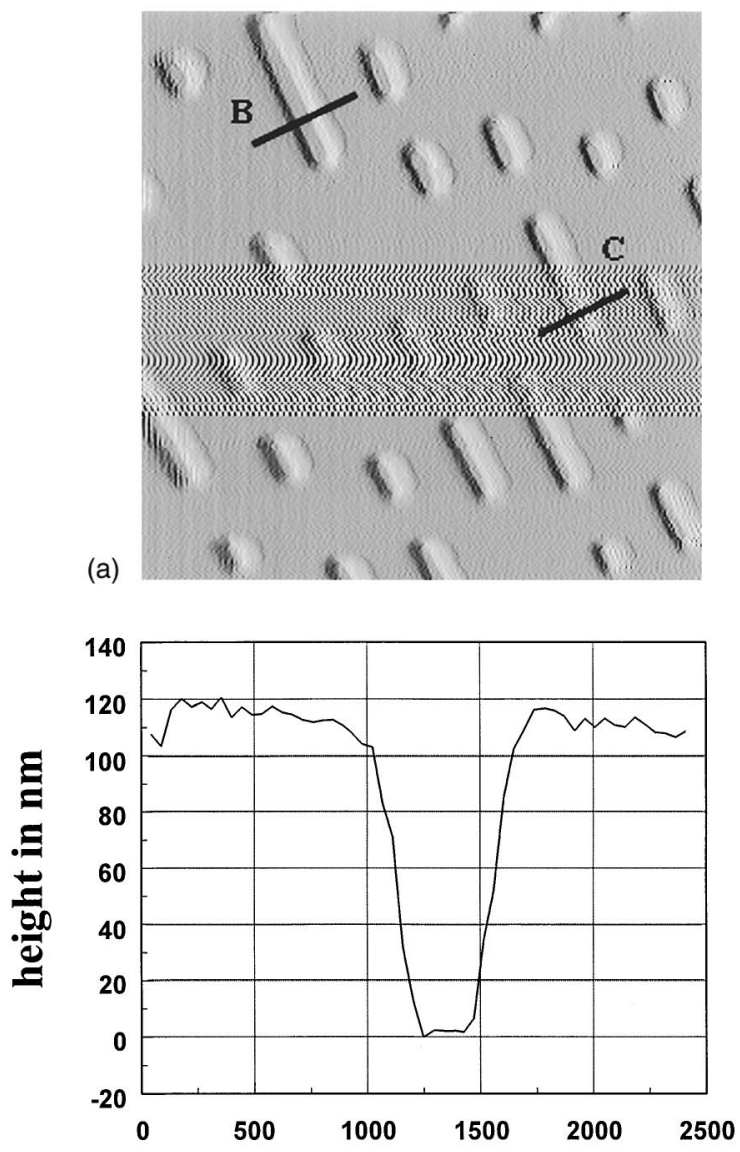

(b)
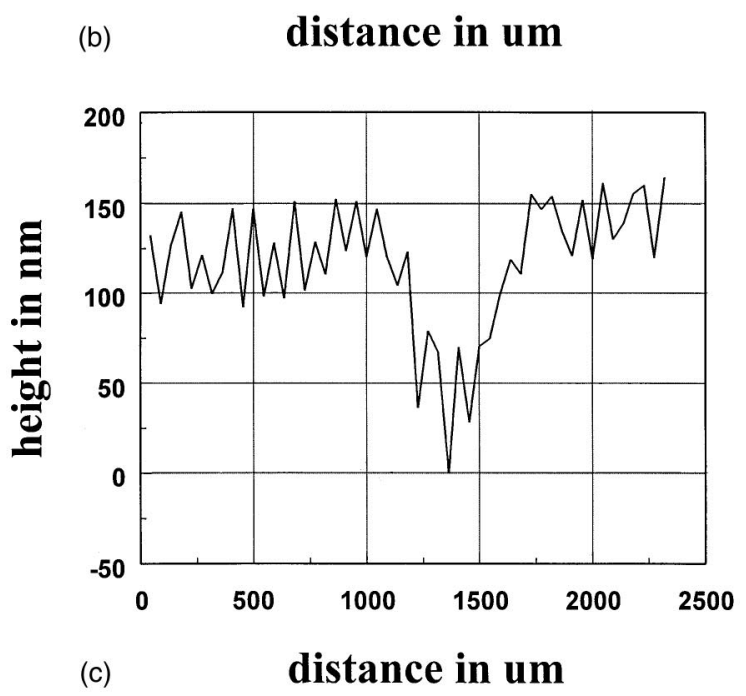

FIG. 4. (A) A $10 \times 10 \mu \mathrm{m}$ AFM image of a CD; GM was initiated at the beginning and end of the scan. (B) When GM is on, the CD pit shape is clearly resolvable. (C) Linecut of a similar pit when GM is off. 

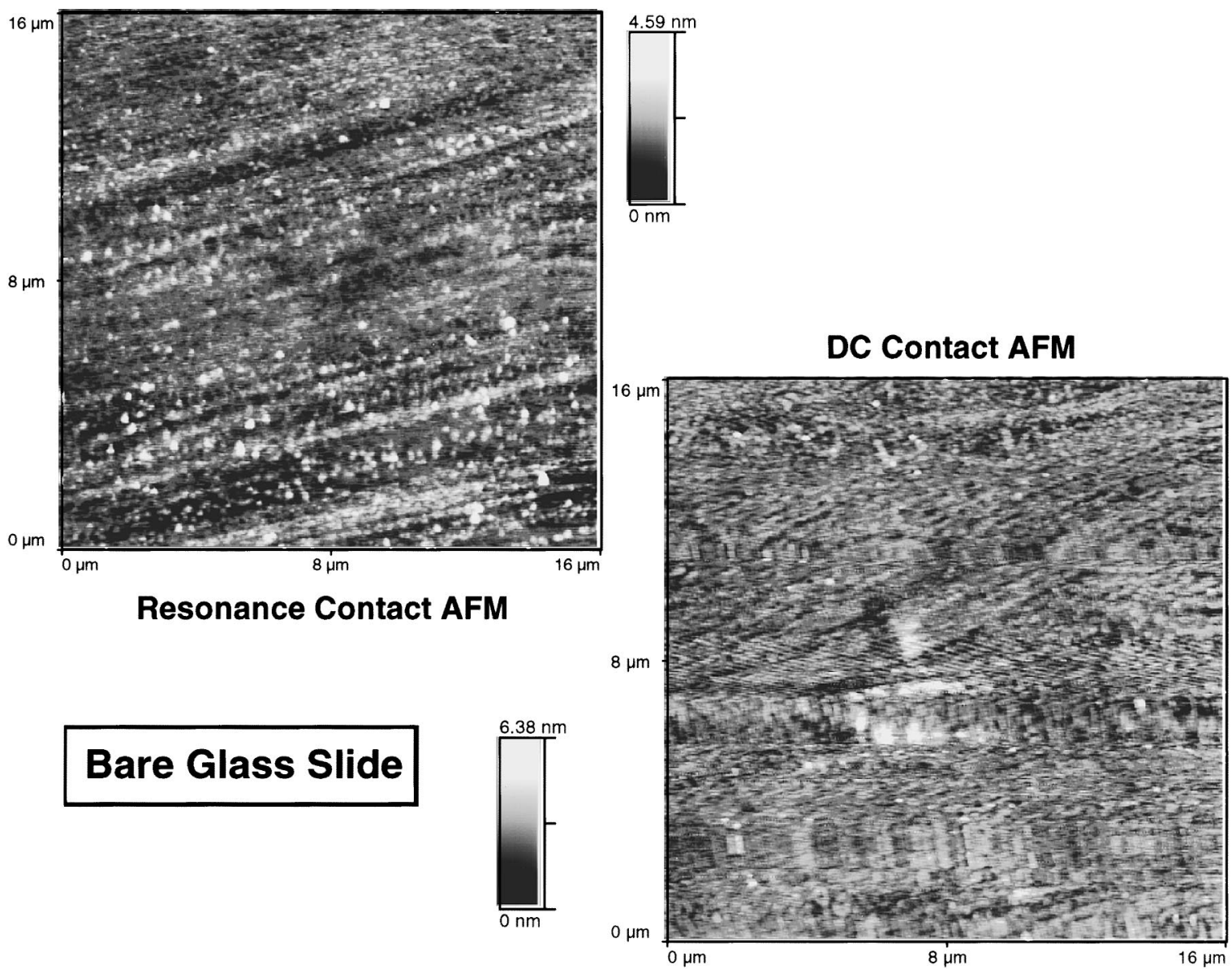

FIG. 5. $16 \times 16 \mu \mathrm{m}$ AFM image of a bare glass slide. In addition to lowering the total noise, GM also decreases the streaking evident in the dc contact image.

technique, a particularly noisy tip-sample system is presented.

Very similar spectra have been obtained with numerous tips and samples. The feedback resonance does not shift appreciably. The mechanical noise shifts from $12-20 \mathrm{kHz}$, depending on the tip, sample, and scanner. Also, GM is effective even when the initial noise is fairly low, for example, 10 $\mathrm{mV}$ of total noise prior to GM can be lowered to the white noise level $(\ll 1 \mathrm{mV})$.

\section{B. Gamble mode}

Figure 3 is a spectrum of the noise in Fig. 2 after GM had been initiated at $356 \mathrm{kHz}$ on GM piezo A. The same results were obtainable when GM piezo B was used at the same frequency. Notice the different vertical scales. The low frequency noise has been lowered by three orders of magnitude. When the initial mechanical noise is less than in Fig. 2 $(<100 \mathrm{mV}$ initial noise), the noise can be totally eliminated (within the detection limits of the spectrum analyzer).

The effectiveness of GM is extremely frequency dependent. Certain GM frequencies have no effect on the noise. An interesting observation is that the noise frequencies can be selectively decoupled; for example, in one experiment, when GM was shifted from 361 to $342 \mathrm{kHz}$, the $13 \mathrm{kHz}$ noise was totally eliminated, but the $3 \mathrm{kHz}$ noise was not altered. Other
GM frequencies lower the amplitude of the high frequency noise while actually raising the amplitude of the low frequency noise.

GM has been successfully implemented with numerous tips, samples, and scanners. The same trends were observed when the meniscus layer was altered from water to oil. GM was also effective when imaging was done completely under water in a standard liquid cell.

\section{Imaging examples}

Figure 4 was taken with an integral gain set $\sim 18$ times higher than optimal. This high feedback gain allowed the image to be acquired very quickly, 12.5 lines per s and 250 pixels per line. The noise values prior to GM were $60 \mathrm{mV}$ at $3 \mathrm{kHz}, 1.0 \mathrm{~V}$ at $15 \mathrm{kHz}$, and $300 \mathrm{mV}$ at $90 \mathrm{kHz}$; with $\mathrm{GM}$ on at $375 \mathrm{kHz}$, there was only a $10 \mathrm{mV}-3 \mathrm{kHz}$ signal. GM was initiated at the beginning and end of the scan and turned off in the middle. Notice the noisy signal between these two points. The linecuts show that the true features of the $\mathrm{CD}$ pit are not resolved without GM. Low frequency noise $(<1$ $\mathrm{kHz}$ ) has also been effectively eliminated while imaging.

Figure 5 is two images of the same area of a bare glass slide. Once GM is initiated, smaller features become apparent. Also, streaking occurs in some images. This streaking has not been observed with GM. 


\section{ACKNOWLEDGMENTS}

S. D. O'Connor is supported by a NIH traineeship. The CIT authors thank Topometrix for the installation and maintenance of the Discoverer SPM system located in their laboratory.

${ }^{1}$ Y. Martin, C. C. Williams, and H. K. Wickramasinghe, J. Appl. Phys. 61, 4723 (1987)
${ }^{2}$ C. A. J. Putman, K. O. Van der Werf, B. G. DeGrooth, N. F. Van Hulst, and J. Greve, Appl. Phys. Lett. 64, 2454 (1994).

${ }^{3}$ P. K. Hansma, J. P. Cleveland, M. Radmacher, D. A. Walters, P. E. Hillner, M. Bezanilla, M. Fritz, D. Vie, H. G. Hansma, C. B. Prater, J. Massie,

L. Fukunaga, J. Gurley, and V. Elings, Appl. Phys. Lett. 64, 1738 (1994).

${ }^{4}$ U. Rabe and W. Arnold, Ann. Phys. 3, 589 (1994).

${ }^{5}$ Topometrix Inc., Santa Clara, CA.

${ }^{6}$ D. R. Baselt, S. M. Clark, M. G. Youngquist, C. F. Spence, and J. D. Baldeschwieler, Rev. Sci. Instrum. 64, 1874 (1993). 